\title{
Control of Plane Non-Holonomic Mobile Robot Based on Image Recogni- tion
}

\author{
Li Xi, Hong Hanyu and Xu Nan ${ }^{*}$
}

School of Electrical and Information Engineering, Wuhan Institute of Technology, 430205, China

\begin{abstract}
With intensive research on robot and wide application of machine vision and movement control in relevant field of robot, image recognition and movement control of robot have become the research focus gradually. This thesis sets the plane non-holonomic mobile robot as the research subject, mainly discusses the control of the plane nonholonomic mobile robot based on image recognition, it has put forward practical stabilization of plane non-holonomic mobile robot based on bounded input, proper controller has been designed aimed at $(2,0)$ type robot and certified feasibility of the controller in theory and simulation; then constructed a test platform for the plane non-holonomic mobile robot which is suitable for this system, introducing this test platform from hardware and software.
\end{abstract}

Keywords: Image recognition, intelligent control, non-holonomic, plane mobile robot.

\section{INTRODUCTION}

In recent years, the mobile robot has been widely used in many different fields including scientific investigation, geologic survey as well as disaster rescue, it has played an important role in discovering profound mystery in universe, conquering the nature as well as understanding our planet. Meanwhile, it could operate under some bad environments such as toxic environment, radiation environment as well as dust environment instead of human beings and has become an indispensable tool. Typical ways of walking of mobile robots are wheel type, crawler type as well as leg and foot type.

Domestic research on mobile robots is later than foreign countries, however, under the support of 863 Scheme, it has obtained many achievements in recent years, in which the most prominent is the underwater robot, the achievement of underwater wireless robot ranks high level worldwide. The fire reconnaissance robot developed by robot institution of Shanghai Jiao Tong University has the functions of explosion-proof and multi-aspect reconnaissance under dangerous, harmful and complex environment. Two kinds of humanoid robots developed by China Automation Science Research Institute, one of them can change hundreds of expressions and the other can paint portraiture for anyone on the spot. "electro-gas welding robot in crawl type" without guide rails and all position developed by Tsinghua University has created a new miracle and has been the pioneer in welding field worldwide, at the same time realized automatic welding of large structural components on site. Mobile robot on ice surface developed by Shenyang Automatic Institute has also accomplished test task successfully in South Pole. This thesis mainly discusses control of plane non-holonomic mobile robot based on image recognition. According to different constraints of plane non-holonomic mobile robot, it can be divided into complete constraint and incomplete constraint, this thesis sets the plane non-holonomic mobile robot as the research subject and makes decisions based on character image recognition, wireless communication as well as sensor to drive the motor realizes intelligent control of robot.

\section{PLANE NON-HOLONOMIC MOBILE ROBOT}

\subsection{With the Development of Automatic Driving Tech- niques}

The mobile robot especially the plane mobile robot that has been widely used most require non-holonomic constraint, that is, non-holonomic constraint of pure rolling without sliding existed between wheels of plane mobile robot and the ground, plane mobile robot cannot make side movement, it is shown as follows:

Wheels of plane non-holonomic mobile robot at any time are only pure rolling but no sliding, its movement direction is only consistent with direction of the robot. This thesis sets plane mobile robot in axial symmetry type in two frontwheel drive with non-holonomic constraint as the research subject, as shown in Fig. (1), conducting research on control of movement of plane non-holonomic mobile robot.

In Fig. (2), the axletrees of two driving wheels of the plane non-holonomic mobile robot coincide and have independent driving system respectively; omni-directional wheel is only the support. To describe movement of the robot, we shall first establish two rectangular coordinate systems, which are overall coordination system $X-Y$ set certain point on two-dimensional surface as the original point and local coordinate system $x_{c}-y_{c}$ which is fixed in forward direction of the lateral axis in body of plane non-holonomic 


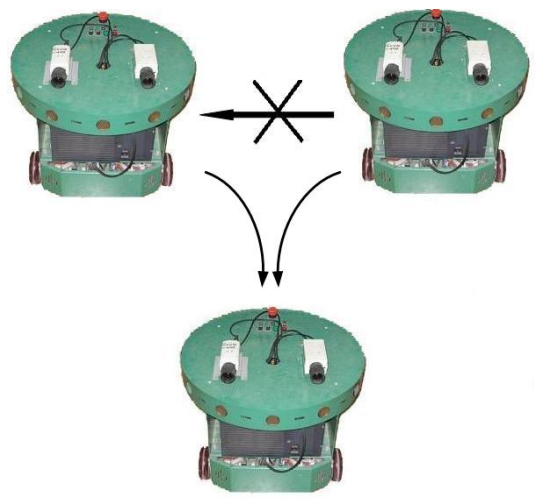

Fig. (1). The plane mobile robot cannot make side movement.

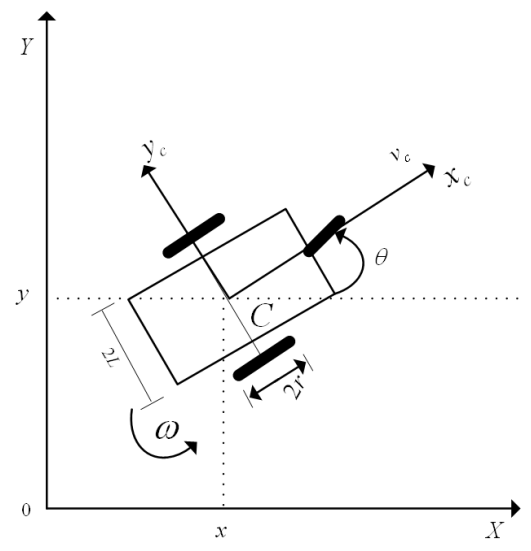

Fig. (2). Model of plane non-holonomic mobile robot.

mobile robot and always has the same direction as it. In which $\mathrm{C}$ is the barycenter of the plane non-holonomic mobile robot, at the same time the original point of local coordinate system. Coordinate of $\mathrm{C}$ under overall coordination system is $(\mathrm{x}, \mathrm{y})$, that is the position of the robot, the included angle $\theta$ of forward directions of $X$ and $x_{c}$ is the orientation of the robot, also called the direction angle.

At present, research on plane non-holonomic mobile robot is divided into kinesiology and dynamics. Kinesiology studies mathematical relation between system location and speed, it could obtain kinesiology model and non-holonomic constraint conditions of plane non-holonomic mobile robot easily with movement analysis method for point particle in physics. While dynamics studies the mathematical relation among system location, speed as well as accelerated speed, which could reflect influence of force on system location and speed including relation between location and speed described in model of kinesiology.

Although model of dynamics is more essential than that of kinesiology, it is more complicated so that the real-time is poorer. And due to some uncertain factors such as friction force, quality as well as moment of inertia, which bring difficulties for the system synthesis, in addition, control rate of kinesiology has basic guidance for design of control rate of dynamics. In view of the above reasons, this thesis has conducted research on kinesiology control of the plane nonholonomic mobile robot.

\subsection{Kinesiology Model of the Plane Non-holonomic Mo- bile Robot}

Kinesiology model of the plane non-holonomic mobile robot describes directly the mathematical relation between the location of system and speed. As it is shown in Fig. (2), the robot is in movement, suppose that there is no slither between wheels and the ground. The relative speed of contact point of wheels and the ground is zero in each instant. Therefore, we could get the constraint condition of the plane non-holonomic mobile robot:

$\dot{x} \sin \theta-\dot{y} \cos \theta=0$

And the kinesiology model deduced from this:

$\left\{\begin{array}{l}\dot{x}=v_{c} \cos \theta \\ \dot{y}=v_{c} \sin \theta \\ \dot{\theta}=\omega\end{array}\right.$ 


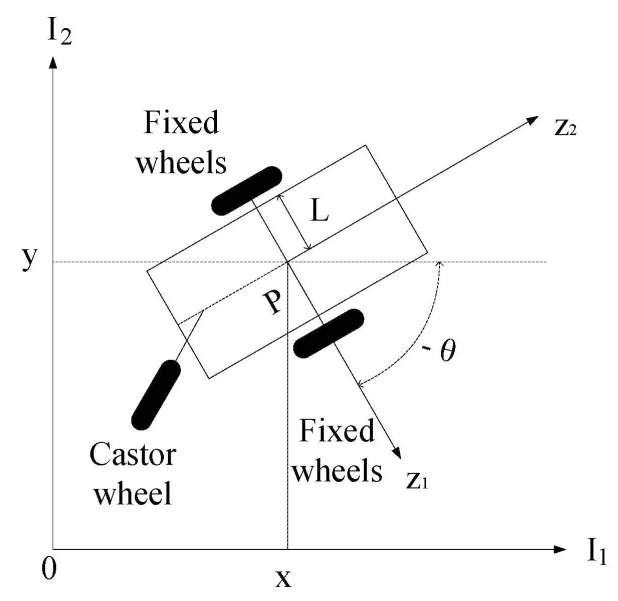

Fig. (3). (2,0) type mobile robot.

In which $(x, y, \theta)$ represents the location of the robot, $v_{c}$ represents the speed of direction of the plane non-holonomic mobile robot that is the speed of $x_{c}$ direction. When the movement direction of the robot is $x_{c}$ forward direction, $x_{c}$ takes the positive value and when the robot moves to $x_{c}$ negative direction, $v_{c}$ takes the negative value. $\omega$ is the speed of steering angle of the plane non-holonomic mobile robot, when the robot turns anticlockwise, $\omega$ takes the positive value and negative value when clockwise.

\section{CONTROL ALGORITHM}

\subsection{Background}

It is well known that stability, maneuverability as well as observed which do not change under change of smooth input and output. If we consider these characters only, we could construct the system easily (for instance, all standard linear systems). Then the design of these controllers becomes so easy. However, for realistic control system, stable and bounded input must be met. Is there any criterion that could make the stability and bounding unchanged under change of input and output? Theoretically, it is a kind of challenging problem, at the same time it has great application potential. What kind of change could ensure the stability and bounding of original system unchanged? It has so many research achievements on linear system [2-4]. For the nonlinear system, there are few methods to stabilize the system with bounded input. A kind of easy method that could solve this problem is only to change the original control system with state transition. Here we put forward a special problem on stability of nonlinear bounded input control system that is the plane non-holonomic mobile robot. In this thesis, we make discussion on stability of plane non-holonomic mobile robot in bounded input.

In realistic control system, to obtain a bounded controller input is of great significance. For a realistic control system, its input may the force, the torque or the voltage. Due to physical and economic limits, these volumes cannot be in- creased without limitation. For instance, control surface, the rudder, flap as well as aileron of the plane are all limited. In this thesis we discuss how to design a bounded input to stabilize the plane non-holonomic mobile robot. Nonholonomic control system is impossible to meet Brockett necessary conditions with smooth pure state feedback. Therefore, we shall only solve this problem with other methods. Control rates for stability available now have three categories: time variation control method [8-10], discontinuous time variation control method $[11,12]$ as well as mixed control method [13, 14], referring to [15] for details.

These three categories of designs of controller have not considered input boundless. That means the input may very large and this kind of controller exists indeed. For instance, for all discontinuous controller inputs may become extremely huge. In documents $[16,17]$, design of controller solves the problem of excessive input. It puts forward to realize the stability of non-holonomic uncertain dynamics of bounded input with the property of chained system. Input of these controllers is based on the bounded input of topological transformation rather than the input of the original system. This would produce many problems in practical application. This thesis puts forward the stability of non-holonomic control system based on practical bounded input. Because the input and output of this new system are the same as that of the original system, bounds of the two are the same.

This thesis considers bounded input stabilization of the plane non-holonomic mobile robot put forward in documents [10] and [19]. As it is shown in Fig. (3), $\xi=[x, y, \theta]^{T}, x, y$ is the coordinate of reference point $\mathrm{P}$ under positive inertial system $\left\{O, I_{1}, I_{2}\right\}, \theta$ is the angular separation on inertial coordinate vector $\left\{I_{1}, I_{2}\right\}$ of base coordinate vector $\left\{z_{1}, z_{2}\right\}$. It is certified that movement of the plane non-holonomic mobile robot can be expressed with integers $\delta_{m}$ and $\delta_{s}$, the plane non-holonomic mobile robot expressed by $\left(\delta_{m}, \delta_{s}\right)$ can be divided into 4 categories:(2,0)type, $(2,1)$ type, $(1,1)$ type as well as $(1,2)$ type. The type used in this thesis is $(2,0)$ type plane non-holonomic mobile robot as shown in Fig. (3). 


\section{2. (2,0) Type Mobile Robot}

For $(2,0)$ type mobile robot, two-step controller can stabilize the system state near any given equilibrium point.

Model for $(2,0)$ type robot is:

$$
\left\{\begin{array}{l}
\dot{x}=-v_{1} \sin \theta \\
\dot{y}=v_{1} \cos \theta \\
\dot{\theta}=v_{2}
\end{array}\right.
$$

The definition of virtual Lyapunov function is as follows:

$V(t)=\frac{1}{2}\left(x^{2}(t)+y^{2}(t)\right)$

Area $\mathrm{D}$ is defined as:

$D=\left\{(x, y, \theta) \mid \sqrt{x^{2}+y^{2}} \leq \sigma, x, y, \theta \in R^{1}\right\}$

Suppose that initial time for the system expressed in formula (3) is $t_{0}=0$ and any given positive number $\varepsilon(0<\varepsilon<1), \sigma, m_{i}(i=1,2)$. The problem that needs to be solved now is how to design the controller $v_{i}(i=1,2)$ to make system (3) meets the condition:

(i) limit time exists $T>0$

$|x(t)| \leq \varepsilon,|y(t)| \leq \varepsilon,|\theta(t)| \leq \varepsilon, t \geq T$

(ii) $\left|v_{i}(t)\right| \leq m_{i}(i=1,2), t \geq 0$

For any initial value $(x(0), y(0), \theta(0)) \in D$

Theorem For system (3) and $\varepsilon(0<\varepsilon<1), \sigma, m_{i}(i=1,2)$ in the above definition, selecting positive numbers $\bar{k}_{1}, k_{1}$ as well as $k_{2}, \alpha: k_{1} \leq \frac{m_{1}}{\sqrt{2 \sigma}}, \sqrt{2} \sigma k_{1}+\bar{k}_{1}<m_{2}, k_{2} \leq m_{2}$, $0<\alpha<\pi$

Then satisfy (i) and (ii) the controller can be realized through the two step as follows:

Step 1 If $\sqrt{V(0)} \leq \varepsilon$ to step 2 directly or take

$v_{1}=-k_{1}(y \cos \theta-x \sin \theta)$,

$v_{2}=v_{1} \frac{x \cos \theta+y \sin \theta}{x^{2}+y^{2}}+\bar{k}_{1} \operatorname{sgn}(e)$

In which $e=-\theta-\alpha+\tan ^{-1}\left(\frac{y}{x}\right)$.

Note that $v_{2}$ in step 1 is defined and it will be certified in specification of theorem.

Then existed limited time $T_{1}$ $\sqrt{V(t)} \leq \varepsilon, t \geq T_{1}$
If $t \geq T_{1}$ turn to the next step.

Step 2 Selecting positive number $k_{2}$ to make $k_{2}\left|\theta\left(T_{1}\right)\right| \leq m_{2}$ . Taking $v_{1}=0, v_{2}=-k_{2} \operatorname{sgn}(\theta)$.

Controller described in the above two steps could make the state and control input of system (3) meets:

(i) Existed limited time $T>0$

$|x(t)| \leq \varepsilon,|y(t)| \leq \varepsilon,|\theta(t)| \leq \varepsilon, t \geq T$.

(ii) $\left|v_{i}(t)\right| \leq m_{i}(i=1,2), t \geq 0$.

For any initial value $(x(0), y(0), \theta(0)) \in D$, $(x(0), y(0), \theta(0)) \in D$.

Proof: we need to do two things, one is transfer conditions of the controller are accomplished in limited time, the other is the controller $v_{i}(i=1,2)$ is bounded, that is, (7) is formed in each step.

Suppose that $(x(0), y(0), \theta(0))$ initial value of any given system (3) in interval D. Obviously, defined from interval D:

$V(0)=x^{2}(0)+y^{2}(0) \leq \sigma^{2}$

Suppose $V(0)>\varepsilon^{2}$. Considering system (3) and formula (9), obtaining the differential of $V(t)$ on $\mathrm{t}$ :

$\dot{V}=(-x \sin \theta+y \cos \theta) v_{1}=-k_{1}(y \cos \theta-x \sin \theta)^{2}$

It is certified that $V(t)$ is digressive, that is $V(t) \leq V(0)$. Therefore, when $V(t) \leq \varepsilon, V(t)>0$ (namely $x^{2}+y^{2}>0$ ). Therefore the first part of $v_{2}$ has been well defined. The second part of $v_{2}$ also requires to be defined.

Due to formula (3), differential of $\tan ^{-1}\left(\frac{y}{x}\right)$ is:

$\frac{d}{d t} \tan ^{-1}\left(\frac{y}{x}\right)=\frac{\dot{y} x-y \dot{x}}{x^{2}+y^{2}}=v_{1} \frac{x \cos \theta+y \sin \theta}{x^{2}+y^{2}}$.

When $V(t) \leq \varepsilon, v_{1}$ is successive and $x^{2}+y^{2}=2 V>0$, therefore $\tan ^{-1}\left(\frac{y}{x}\right)$ has the definition. In addition, $e$ has the definition in step 2 , therefore $v_{2}$ has been defined.

Due to formula (3), differential of $e$ on $\mathrm{t}$ is:

$\dot{e}=-v_{2}+\frac{\dot{y} x-y \dot{x}}{x^{2}+y^{2}}=-v_{2}+v_{1} \frac{x \cos \theta+y \sin \theta}{x^{2}+y^{2}}$

Taking $v_{2}$ in formula (9) into

$\dot{e}=-k_{1} \operatorname{sgn}(e)$ 


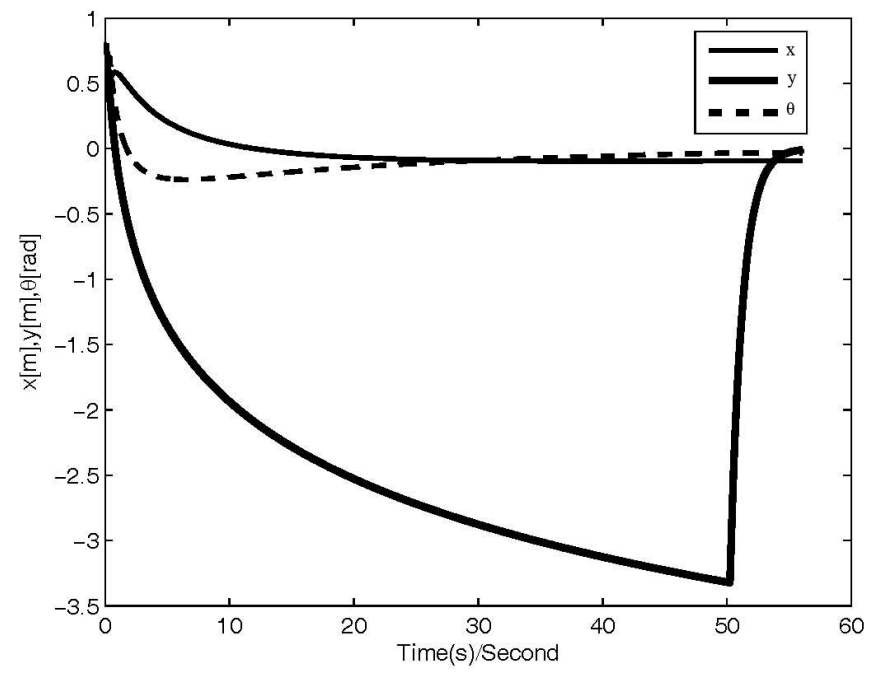

Fig. (4). Loci of $x(t), y(t), \theta(t)$.

According to VSCT, existed limited time $T_{0}>0$ to make $e \equiv 0, t>T_{0}$

$-\theta+\tan ^{-1}\left(\frac{y}{x}\right) \equiv \alpha, t>T_{0}$

$|y \cos \theta-x \sin \theta|=\sqrt{x^{2}+y^{2}}\left|\sin \left(-\theta+\tan ^{-1}\left(\frac{y}{x}\right)\right)\right|$

Obtaining from formula (11) and definition of $V$ : $\dot{V}=-2 k_{2} V \sin ^{2}\left(-\theta+\tan ^{-1}\left(\frac{y}{x}\right)\right)$.

Taking into formula (13): $\dot{V}=-2 k_{2} V \sin ^{2} \alpha, t>T_{0}$

Due to $\sin ^{2}(\alpha)>0$, therefore index number of $V(t)$ tends to 0 , this shows it could select one limit time $T_{1}\left(T_{1} \geq T_{0}\right)$ to make $V(t) \leq \varepsilon^{2}, t \geq T_{1}$

This shows condition of transfer to step 2 can be obtained.

According to formula (9) and (8), it could deduce within $T_{1} \leq t \leq T_{2}$

$$
\begin{aligned}
\left|v_{1}(t)\right| & =\left|k_{1}(y \cos \theta-x \sin \theta)\right| \\
& =\left|k_{1} \sqrt{x^{2}+y^{2}} \sin \left(-\theta+\tan ^{-1}\left(\frac{y}{x}\right)\right)\right| \\
& \leq k_{1} \sqrt{2 V(t)} \leq k_{1} \sqrt{2 V(0)} \leq k_{1} \sqrt{2} \sigma \leq m_{1} .
\end{aligned}
$$

According to formula (9), it could deduce within
$T_{1} \leq t \leq T_{2}:\left|v_{2}\right|=\left|\left(v_{1} \frac{x \cos \theta+y \sin \theta}{x^{2}+y^{2}}+\bar{k}_{1} \operatorname{sgn}(e)\right)\right| \leq\left|v_{1}\right|+\bar{k}_{1}$.

According to previous result and formula (8), we could get $\left|v_{2}\right| \leq \sqrt{2} \sigma k_{1}+\bar{k}_{1} \leq m_{2}, T_{1} \leq t \leq T_{2}$

We will prove step 2 in as follows:

Since $v_{1} \equiv 0$, we could get according to system (1): $y(t)=y\left(T_{1}\right), x(t)=x\left(T_{1}\right), t \geq T_{1}$

On the other hand, we could see clearly from system 1 that $\dot{\theta}=-k_{2} \operatorname{sgn}(\theta), t \geq T_{1}$

According to VSCT, existed limited time $T_{2}>0$, when $t \geq T_{2}\left(T_{2}>T_{1}\right), \theta(t) \equiv 0$. Obviously $\left|v_{1}\right| \leq m_{1}$ in step 2 . According to the definition of $k_{2}$ in step 2, $\left|v_{2}\right| \leq m_{2}$ has formed.

Therefore taking the time $T=T_{2}>0$, then when $t \geq T$, $|x(t)| \leq \varepsilon,|y(t)| \leq \varepsilon,|\theta(t)| \leq \varepsilon$. During the whole control process, $\left|v_{i}\right| \leq m_{i},(i=1,2)$, this has proved theorem 1 .

\subsection{Simulation Result}

For robot model in $(2,0)$ type, taking $m_{1}=4, m_{2}=7$, $\alpha=\pi / 2, \sigma=1$, according to calculation of formula (3.12), $k=1, k_{1}=4, k_{2}=2$. Taking $\varepsilon=0.01$, initial value is $x(0)=[0.5,0.7,0.8]$. Figs $(\mathbf{4}, \mathbf{5}$ and $\mathbf{6})$ are position coordinates $x(t), y(t), \theta(t)$ respectively, linear velocity and angular 


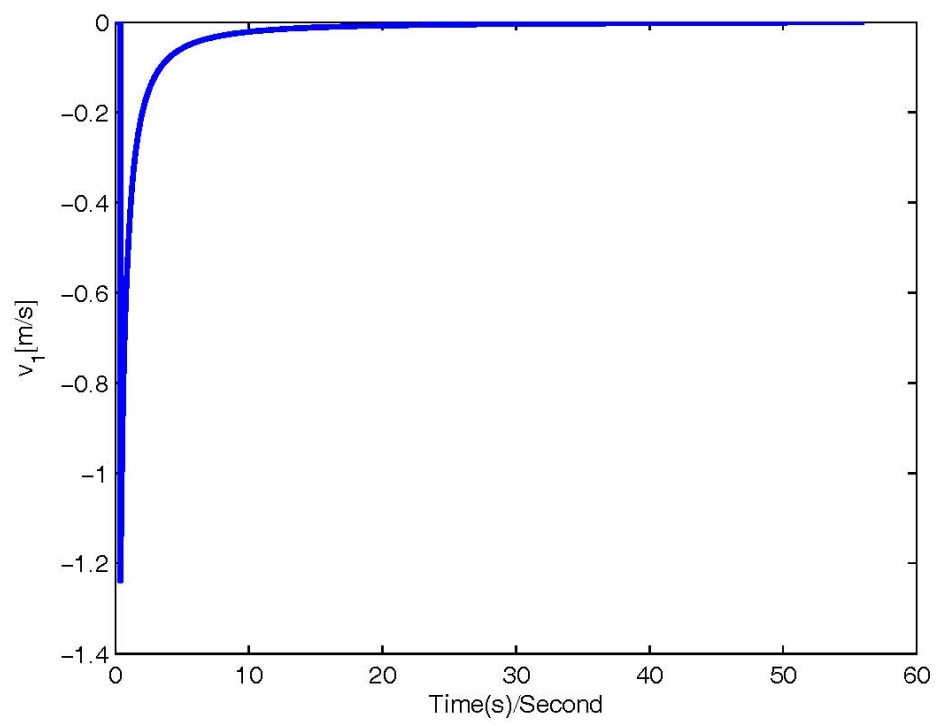

Fig. (5). Locus of linear velocity $v_{1}(t)$.

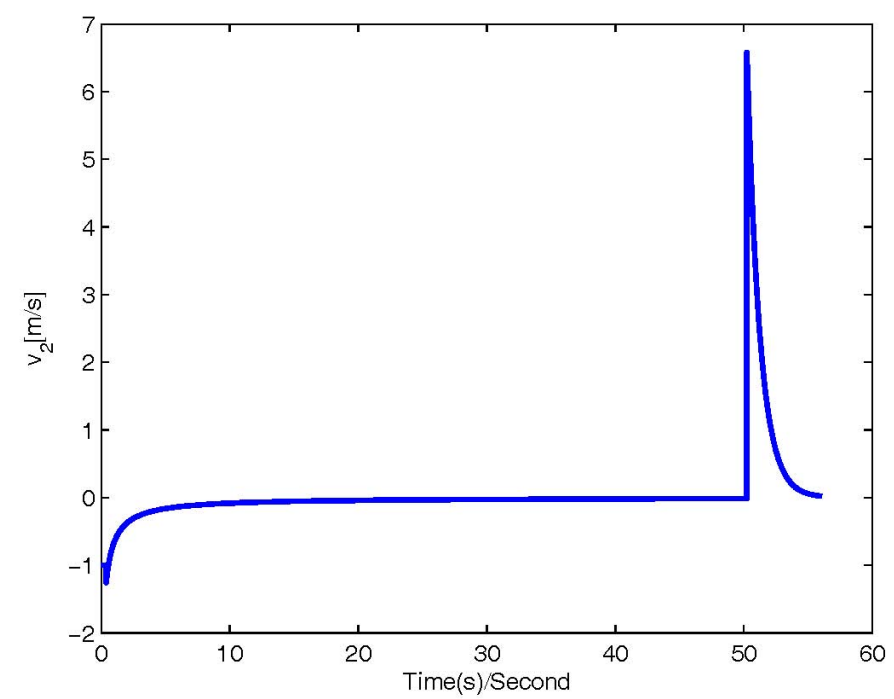

Fig. (6). Locus of angular velocity $v_{2}(t)$.

velocity. It is known from simulation diagram that position coordinates are convergent and the input is also within the scope defined.

\section{DESIGN AND TEST OF SYSTEM SOFTWARE}

\subsection{Design of System Software}

AS-UII robot is a new generation of intelligent horizontal mobile robot facing the education. AS-UII robot owns a powerful micro processing system and perfect sensor system and it can expands to auditory sense, vision as well as sense of touch to be a real intelligent robot.

Software development environment for AS-UII robot is an open software development platform which is suitable for plane non-holonomic mobile robot, it has integrated programming environment of many aspects including environ- mental perception, task planning, wireless communication, behavior control as well as behavior selection, it is compatible with various kinds of sensors such as infrared encoder and photoelectric encoder and supports various kinds of designs and developments of motion control.

AS-UII robot software provides a large amount of sensor variables while provides various kinds of interface functions including serial communication and wireless communication. It could accomplish many kinds of complex tasks well including robot navigation, robot tracking as well as team cooperation.

In addition, it can expand in route planning, creation of tasks as well as behavior control according to our personal requirements.

In this test, the programming language used by AS-UII robot is graphical and interactive C language(VJC for short), 


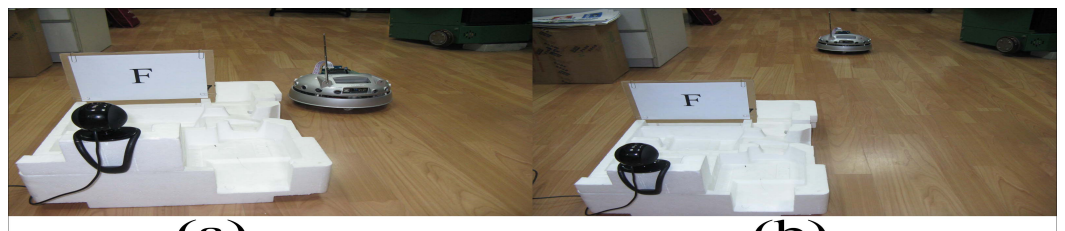

(a)

(b)
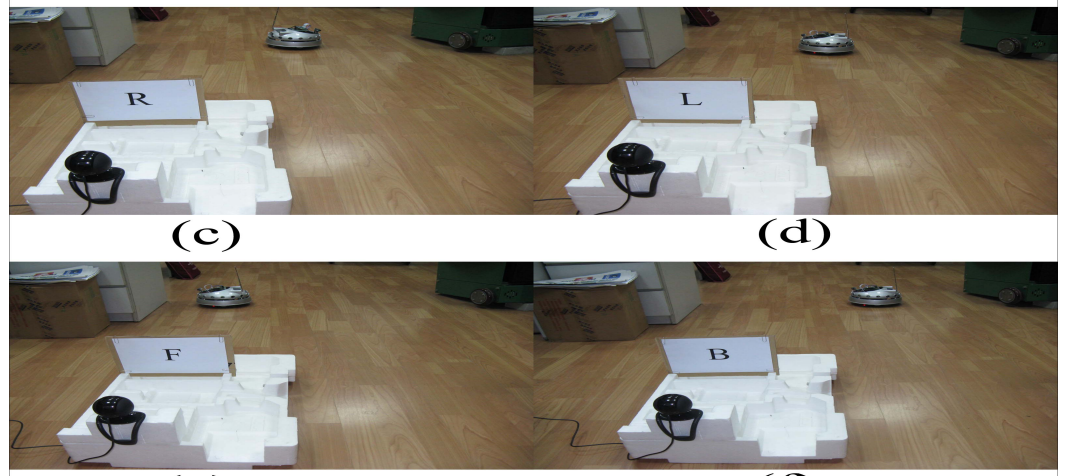

(e)

(f)

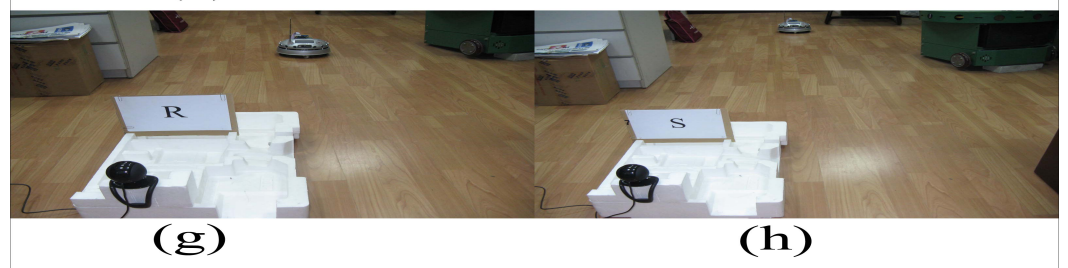

Fig. (7). (a) Initial position, (b) Forward, (c) Turn right, (d) Turn left, (e) Obstacle in forwarding and auto stop, (f) Backward, (g) Turn right, (h) Stop.

it is a kind of special software development system of ASUII robot. VJC1.6 development version can be operated on 95/98 and Windows NT 4.0 and above. VJC1.6 development version is composed by graphical programming interface and JC code programming interface to edit, compile as well as link to the program, then download into AS-UII robot through serial port.

Type of organization of AS-UII robot software adopts hierarchy architecture, it is divided into 3 layers, they are sensing layer, task layer as well as behavior layer respectively. Among which the sensing layer is at the bottom, task layer and behavior layer are on the upper layer. In the sensing layer, this development platform provides functions such as communication interface to the sensor, sensor data processing as well as remote communication interface including various kinds of sensor communication interfaces such as infrared encoder and photoelectric encoder.

\subsection{Test}

This test environment is the upper monitor and AS-UII robot, the upper monitor is used to conduct character recognition and give recognition result, AS-UII robot makes analysis and decisions and accomplishes a series of actions according to recognition result and independent various sensor information, the two are connected through serial port and radio wireless communication.

The upper monitor is a kind of common PC, CPU is Intel Core2 E4600, 2G internal memory, the operating system is Windows XP; Logitech QuickCam camera is used to collect images 1.3 million pixel CCD, USB2.0 interface; one wireless transmission module based on RS-232 serial port. One infrared distance sensor, two infrared measuring sensor, collision detection sensor as well as one wireless transmission module have been installed on AS-UII robot.

The upper monitor needs to recognize pictures marked $\mathrm{F}$, B, L, R, S read from the camera, these 5 letters represent forward, backward, turn left, turn right as well as stop respectively. The recognition result will be transmitted to the robot through wireless communication module by the end of recognition. When the robot receives the signal sent by the upper monitor, it will decide the action required to execute according to recognition result and sensor information including infrared distance measurement.

\subsection{Test Result}

According to a large amount of tests, we have summarized that the motor value of AS-UII robot in going forward is $(20,23)$ and 10 for turning left, $(-15,-16)$ for going backward, it is rational for measuring the distance threshold is 40 . The test result has been given in Fig. (7).

It can be seen from the figure that AS-UII robot has accomplished preset actions basically according to the requirement and has a series of functions such as obstacle avoidance and distance measurement. Of course, it has accomplished some easy actions at present, the control strategy has not been so good and the function not so strong, many functions of the robot in control and entertainment have yet to be further developed and improved. 


\section{CONCLUSION}

This thesis has fully discussed the control method of plane non-holonomic mobile robot based on image recognition. It sets the plane non-holonomic mobile robot as the research subject, focusing on controlling the robot based on recognition of character image and putting forward a new kind of method of character image recognition to make the robot action according to figures. It has certified effectiveness of new algorithm of character image recognition. It has also certified the effectiveness of control method of robot theoretically under rational assumption; simulation experiment and system experiment have certified effect of control method well. The thesis puts forward the control algorithm based on $(2,0)$ type robot system and certifies theoretically that the control method could stabilize the system gradually and certifies the effectiveness of this method in simulation experiment. Interconnection based on PC and AS-UII robot platform has realized to control the robot with image, certifying feasibility of this assumption, credibility of character image recognition as well as effectiveness of control algorithm through the experiment.

\section{CONFLICT OF INTEREST}

The authors confirm that this article content has no conflict of interest.

\section{ACKNOWLEDGEMENTS}

This work is supported by the National Science Foundation of China under Grant 61175013. It is also supported by the National Science Foundation of Hubei Province under Grant 2012FFA046 and the International Cooperation Plan Project of Wuhan City under Grant 2014030709020310.

\section{REFERENCES}

[1] Y. Mei, Vision Feedback Stabilization of Non-holonomic Mobile Robot, Master's thesis Shanghai University of Technology, 2009.

[2] J. Solís-Daun, J. Álvarez - Ramírez, and R. Suárez, "Semiglobal stabilization of linear systems: a parametric optimization approach," Robust Nonlinear Control, vol. 9, no. 8, pp. 461-484, 1999.

[3] R. Suárez, J. Álvarez - Ramírez, and J. Solís-Daun, "Linear systems with bounded inputs: global stabilization with eigenvalue placement," International Journal of Robust Nonlinear Control, vol. 7, no. 9, pp. 835-845, 1997.
[4] H.J. Sussmann, E.D. Sontag, and Y. Yang, "A general result on the stabilization of linear systems using bounded controls," IEEE Transactions on Automatic Control, vol. 39, no. 12, pp. 2411-2424, 1994.

[5] Y. Lin and E.D. Songtag, "A universal formula for stabilization with bounded controls," Systems Control Letters, vol. 16, no. 6, pp. 393-397, 1991.

[6] R.W. Brockett, "Asymptotic stability and feedback stabilization," Differential Geometric Control Theory, Birkhauser, vol. 27, pp. 181-208, 1983.

[7] D. Aeyels, "Stabilization of a class of nonlinear systems by smooth feedback," System and Control Letters, vol. 24, no. 5, pp. 289-294, 1985.

[8] J.P. Pomet, "Explicit design of time varying stabilizing control laws for a class of controllable systems without drift," Systems and Control Letters, vol. 18, no. 2, pp. 147-158, 1992.

[9] J.M. Coron, "Global asymptotic stabilization for controllable systems without drift," Mathematics of Control, Signals and Systems, vol. 5, no. 3, pp. 295-312, 1992.

[10] A. Teel, R. Murray, and G. Walsh, "Non-holonomic control systems: From steering to stabilization with sinusoids," In: Proceedings of $31^{\text {st }}$ IEEE Conference on Decision and Control, 1992, pp. 1603-1609.

[11] C.C. de Wit and O.J. Sordalen, "Exponential stabilization of mobile robots with non-holonomic constraints," IEEE Transactions on Automatic Control, vol. 37, no. 11, pp. 1791-1797, 1992.

[12] A.M. Bloch, and S. Drakunov, "Stabilization of a non-holonomic systems via sliding modes," In: Proceedings of the IEEE Conference Decision and Control, 1994, pp. 2961-2963.

[13] O.J. Sordalen, and O. Egeland, "Exponential stabilization of nonholonomic chained systems," IEEE Transaction on Automatic Control, vol. 40, no. 1, pp. 35-49, 1995.

[14] Kolmanovsky, M. Reyhanoglu, and N.H. McClamroch, "Switched mode feedback control laws for non-holonomic systems in extended power form," Systems and Control Letters, vol. 27, no. 1, pp. 29-36, 1996.

[15] H.Y. Kolmanovsky, and N.H. McClamroch, "Development in nonholonomic control problems," IEEE Control Systems, vol. 15, no. 6, pp. 20-36, 1995.

[16] P. Tsiotras, and J. Luo, "A reduced-effort control law for underacted rigid spacecraft," Journal of Guidance, Control, and Dynamic, vol. 20, pp. 1089-1095, 1997.

[17] J. Luo, and P. Tsiotras, "Control design for systems in chained form with bounded inputs," In: American Control Conference, Philadelphia, PA, 1998, pp. 473-477.

[18] C.L. Wang, W. Huo, D. Tan, and Y. Wang, "Stabilization of uncertain non-holonomic dynamic systems with bounded inputs," Control Theory and Application, vol. 17, no. 6, pp. 831-835, 2000.

[19] G. Campoin, G. Bastin, and B. Dandrea-Novel, "Structure properties and classification of kinematic and dynamic models of wheeled mobile robots," IEEE Transactions on Robotics and Automation, vol. 12, no. 1, pp. 47-62, 1996.

[20] J. Du, and C. Wang, "Practical stabilization of non-holonomic wheeled mobile robot systems," In: Proceedings of the $7^{\text {th }}$ Asian Control Conference, 2009, pp. 1503-1508.

(C) Xi et al.; Licensee Bentham Open.

This is an open access article licensed under the terms of the Creative Commons Attribution Non-Commercial License (http://creativecommons.org/licenses/by-nc/3.0/) which permits unrestricted, non-commercial use, distribution and reproduction in any medium, provided the work is properly cited. 